\title{
The new system of family Woodsiaceae
}

\section{Новая система семейства Woodsiaceae}

\author{
A.I. Shmakov \\ А.И. Шмаков \\ South-Siberian Botanical Garden, Altai State University, Lenina str., 61, Barnaul, 656049, Russia \\ Южно-Сибирский ботанический сад, Алтайский государственный университет, \\ np-m Ленина, 61, Барнаул, 656049, Россия._E-mail: alex_shmakov@mail.ru
}

\begin{abstract}
Key words: Woodsia, new system, Eriosoriopsis, Protowoodsia, Hymenocystis, Cheilanthopsis, Physematium, Woodsiopsis.
\end{abstract}

Ключевые слова: Woodsia, новая система, Eriosoriopsis, Protowoodsia, Hymenocystis, Cheilanthopsis, Physematium, Woodsiopsis.

Summary. A new system of the family Woodsiaceae is proposed. A new genus Woodsiopsis is described and new combinations in the genera Physematium, Eriosoriopsis, and Woodsiopsis are validated. The systems of Woodsia and Eriosoriopsis are clarified and new intrageneric taxa in these genera are established.

Резюме. Предложена новая система семейства Woodsiaceae. Описан новый род Woodsiopsis, обнародованы новые комбинации в родах Physematium, Eriosoriopsis и Woodsiopsis. Уточнены системы родов Woodsia и Eriosoriopsis, в которых описаны новые внутриродовые таксоны.

The family Woodsiaceae (Diels) Herter which is accepted by the present (Shmakov, 1999, 2001, 2003, 2009a, b, 2011; Shmakov, Kiselev, 1995) and some other authors (Tzvelev, 1991, 2005; Christenhusz, Zhang, Schneider, 2011; Rothfels et al., 2012; Zhang, Kato et Shmakov, 2013) in the narrow sense, is continuously being in focus of many ptegidologists. A narrow taxonomic concept of the family is favored by molecular studies of two last decades (Rothfels et al., 2012). However, Woodsiaceae is still rather heterogenic morphologically (e. i., it includes taxa with and without stipe articulation, with different shape and dissection of indusia, etc.). Molecular phylogenetic studies of Woodsia s. 1. reflects well this fact and demonstrate rather deep split between the Old World and predominantly New World clades (Rothfels et al., 2012; Larsson, 2014).
Having the above characters in mind, we consider it meaningful to divide Woodsiaceae onto two subfamilies (Shmakov, 2001): Woodsioideae Schmakov (stipe articulate or continuous, indusia membranous and marginal or divided into filiform segments) and Protowoodsioideae Schmakov (stipes continuous, indusia sphaerical or lobed). The first subfamily is widely distributed in Northern Hemisphere (Eurasia and North America) and comprises two genera (Woodsia R. Br., Eriosoriopsis (Kitag.) Ching \& S. H. Wu). The second subfamily is represented by five genera (Protowoodsia Ching, Hymenocystis C. A. Mey., Cheilanthopsis Hieron., Physematium Kaulf., Woodsiopsis Schmakov) distributed primarily in the New World with fewer species in Africa, Madagascar, Caucasus, and East Asia.

Woodsia is the most difficult genus of the family with respect to both systematics and understanding its evolutionary history. Taxonomically valuable characters are degree of dissection and indumentum (including hairs and scales) of fronds as well as the form (oblique of almost perpendicular) and location (above, below, or near the middle of stipe) of the articulation. These characters were used by a number of authors (Nakai, 1925; Ching, 1932; Brown, 1964; Ma, 1985; Shmakov, Kiselev, 1995; Shmakov, 2001; Tzvelev, 2005) for intrageneric classification of Woodsia. The present system is also based on the 
characters of fronds (dissection, indumentum) and stipe articulation (form, location).

The new system of Woodsiaceae and some of its genera is presented below.

Protowoodsioideae Shmakov, 2001, Turczaninowia, 4 (1-2): 65. - Stipes continuous, indusia spherical or divided into lobes.

Type: Protowoodsia Ching

Five genera and about 23 species distributed mainly in the New World, fewer ones in Africa, Madagascar, the Caucasus and East Asia.

Protowoodsia Ching, 1945, Lingn. Sci. J. 21, 1-4: 36.

Type: P. manchuriensis (Hook.) Ching

One species in subtropical and partly temporate areas of East Asia.

Hymenocystis C. A. Mey, 1831, Verzeichn. P1. Cauc: 229. - Physematium sect. Hymenocystis (C. A. Mey.) Tzvel., 2005, Novit. Syst. Pl. Vasc., 37: 34.

Type: H. fragilis (Trev.) A. Askerov (= H. caucasica C. A. Mey.)

One species in Caucasus.

Cheilanthopsis Hieron., 1920, Notizbl. Bot. Gard. Berlin-Dahlem, 7. 69: 409.

Type: C. indusiosa (H. Christ) Ching

Three species in Hymalaya and SW China (C. indusiosa (H. Christ) Ching, C. elongata (Hook.) Copel., C. kangdingensis (H. S. Kung, L. B. Zhang \& X. S. Guo) Shmakov).

Physematium Kaulf. 1829, Flora 12: 341.

Type: P. molle Kaulf.

Five species distributed in Central and South America, Africa and Madagascar.

Physematium angolensis (Schelpe) Shmakov, comb. nov. - Woodsia angolensis Schelpe, 1976, in Garcia de Orta, Sér. Bot. 3: 53. - Holotype: "Angola, Huíla, Lubango, Tundavala (Serra da Chela) ao quilòmetro 18 da escarpa rochosa junto à fends, 30 IV 1971 , A. Borges 131" (holotype - LISC; isotypes - COI, LUAI).

Physematium burgessiana (Gerr. ex Hook. \& Baker) Shmakov, comb. nov. - Woodsia burgessiana Gerr. ex Hook. et Baker, 1866, Synopsis filicum, 2: 48. - Woodsia montividensis (Spreng.) Hieron. var. burgessiana (Gerr. ex Hook. et Baker) Schelpe, 1969, J. S. Afr. Bot. 35: 138. - Type: "Natal, near the Tugela River, Gerrard \& McKen s.n." (holotype - K 000351038; isotypes - S, SAM, TCD).
Physematium canescens (Kunze) Trevis., 1875, Nuovo Giorn. Bot. Ital. 7: 155. - Woodsia canescens (Kunze) Mett., 1864, Ann. Sci. Nat. Bot, sér. 5, 2: 249, Fig. 324L. - Cheilanthes canescens Kunze, 1839, Linnaea 13: 143.

Physematium molle Kaulf., 1829, Flora 12: 341.

Physematium montividensis (Spreng.) Shmakov, comb. nov. - Woodsia montividensis (Spreng.) Hieron. 1896, Bot. Jahrb. 22: 363. - Dicksonia montevidensis Spreng., 1827, Syst. Veg., ed. 16, 4, 1: 122. - Type: "Monte Video, Sello 517" (B).

Woodsiopsis Schmakov, gen. nov. - Woodsia subgen. Perrinia Hook. 1844, Sp. Fil. 1: 62, p.p. Cespitose plants. Rhizomes short or creeping, erect or horizontal. Scales bicolor, with dark central stripe and pale brown margins. Stipes continuous. Indusium dissected almost to the base onto the blades or narrow, usually filiform segments. The basic chromosome number $\mathrm{n}=38$.

Type: W. obtusa (Spreng.) Shmakov (Woodsia obtusa (Spreng.) Torrey)

10 species and 3 subspecies distributed in North America and Mexico.

Woodsiopsis appalachiana (T. M. C. Taylor) Shmakov, comb. nov. - Woodsia appalachiana T. M. C. Taylor, 1947, Amer. Fern J. 37: 88. - Holotype: "USA. West Virginia: on a mountain 4 miles north of Old Sweet, 14 IX 1903, Steele \& Steele 306" (holotype - GH; isotype - MO).

Woodsiopsis cochisensis (Windham) Shmakov, comb. nov. - Woodsia cochisensis Windham, 1993, Contr. Univ. Michigan Herb. 19: 54, fig. 7. Holotype: "USA. Arizona: Cochise Co., SE wall of Huachuca Canyon in the Huachuca Mts., ca. 2.85 km SE of Blacktail Spring, $6000 \mathrm{ft}, 31$ VIII 1985, M.D. Windham (781) \& G. Yatskievych" (holotypeUT; isotypes - ARIZ, ASU, MICH, UC, US).

Woodsiopsis cystopteroides (Windham et Mickel) Shmakov, comb. nov. - Woodsia cystopteroides Windham et Mickel, 2004, Mem. New York Bot. Gard. 88: 688, fig. 325 F-H, J-K. - Type: "Mexico. Sinaloa: Ocurahui, Sierra Surotato. Pine Forest, 6000-7001 ft.; igneous rocky slope in open PineOak forest, 1-10 IX 1941 , Gentry 6432" (holotype $\mathrm{NY}$; isotypes - ARIZ, GH, MICH, PH).

Woodsiopsis mexicana (Fée) Shmakov, comb. nov. - Woodsia mexicana Fée, 1857, Sept. Mem. Fam. Foug.: 66. - Type: "Mexico, San Angel, 1855, W. Schaffner 306 (?)".

Woodsiopsis neomexicana (Windham) Shmakov, comb. nov. - Woodsia neomexicana Windham, 1993, Contr. Univ. Michigan Herb. 19: 52, fig. 
6. - Type: "USA. New Mexico: Socorro Co., along small tributary of Water Canyon in the Magdalena Mountains ca. $5.84 \mathrm{~km} \mathrm{SE}$ of the summit of North Baldy, 7050 ft, 23 VIII 1990, M.D. Windham (90365) \& E. Rahe" (holotype - UT; isotypes - ARIZ, ASU, BRY, COLO, GH, MICH, MO, NMC, NY, TEX, UC, UNM, US).

Woodsiopsis obtusa (Spreng.) Shmakov, comb. nov. - Woodsia obtusa (Spreng.) Torr., 1840, New York State, Rep. Geol. Surv. 195. - Polypodium obtusum Sprengel, 1804, Anleit. Kenntn. Gew. 3: 93. Type: “America septentr. Leg.: M. Kinn s.n." (B!).

Woodsiopsis obtusa subsp. occidentalis (Windham) Shmakov, comb. nov. - W. obtusa subsp. occidentalis Windham, 1993, Contr. Univ. Michigan Herb. 19: 56. - Type: "USA. Texas: Llano Co., W side of Inks Lake, on hillside in granite area, 16 IV 1945, Lundell B484" (holotype - LL!; isotypes LL! RM!).

Woodsiopsis oregana (D. C. Eaton) Shmakov, comb. nov. - Woodsia oregana D. C. Eaton, 1865, Canad. Naturalist \& Quart. J. Sci. n. s. 2: 90. - Type: "USA. Oregon: Dalles of the Columbia River, Major Raines in 1855" (lectotype - YU; isolectotypes $\mathrm{GH}, \mathrm{K})$.

Woodsiopsis oregana subsp. cathcartiana (B. L. Rob.) Shmakov, comb. nov. - Woodsia oregana subsp. cathcartiana (B. L. Rob.) Windham, 1933, Contr. Univ. Michigan Herb. 19: 58. - Woodsia cathcartiana B. L. Rob., 1908, Rhodora 10: 30. - Type: "USA. Minnesota: Taylor's Falls of the St. Croix River, 1874, Cathcart s.n." (holotype $\mathrm{GH} !)$.

Woodsiopsis phillipsii (Windham) Shmakov, comb. nov. - Woodsia phillipsii Windham, 1993, Contr. Univ. Michigan Herb. 19: 50, fig. 5. - Type: "USA. Arizona. Cochise Co.: Rucker Canyon, Chiricahua Mountains, canyon sides in pine woods, 6500 feet, 7 X 1945, Walter S. Phillips, 2854" (holotype - GH; isotypes - ARIZ, ASC, UBC, US).

Woodsiopsis plummerae (Lemmon) Shmakov, comb. nov. - Woodsia plummerae Lemmon, 1882, Bot. Gaz. 7: 6. - Type: "USA: Arizona: Chiricahua Mts., 24 Sept 1881, Lemmon \& Lemmon, 891" (RSA; UC; IT: F, GH, K, US).

Woodsiopsis scopulina (D. C. Eaton) Shmakov, comb. nov. - Woodsia scopulina D. C. Eaton, 1865, Canad. Naturalist \& Quart. J. Sci. 2: 91. - Type: "North America. USA. Colorado. Middle Park. C. C. Parry. 1861" (Syntype - YU); "USA. Flora Montium Scopulosorum, sub. lat. 39-41 ${ }^{\circ}$, Hall E. \& Harbour J. P., 690b, 1862” (isosyntypes - UC, F).

Woodsiopsis scopulina subsp. laurentiana (Windham) Shmakov, comb. nov. - Woodsia scopulina subsp. laurentiana Windham, 1993, Contr. Univ. Michigan Herb. 19: 59. - Type: "Canada. Quebec: Gaspe Co., Tourelle, on sandstone seacliffs, 19-21 VIII 1905, Collins \& Fernald 25351" (holotype - GH!; isotypes - CAN! CAS! GA! GH! MICH! NY! POM! UC! US!).

Woodsioideae Shmakov, 2001, Turczaninowia, 4 (1-2): 65. - Stipes articulated or continuous. Indusium plate-like, dissected onto unequal parts or pinnatisect with filiform segments.

Type: Woodsia R. Br.

Two genera and about 36 species distributed mainly in Eurasia with few representatives in the north of North America.

Woodsia R. Br., 1810, Prodr. Fl. Nov. Holl.: 158. Type: $W$. ilvensis (L.) R. Br.

About 28 species almost worldwide.

Subgen. Acrolysis (Nakai) Shmakov, 2003, Pteridol. New Millennium: 52. - Sect. Acrolysis Nakai, 1925, Bot. Mag. Tokyo, 39: 176.

Lectotype: $W$. polystichoides D. C. Eaton

Stipe with an oblique articulation.

Sect. Acrolysis Nakai, 1925, Bot. Mag. Tokyo, 39: 176. - Sect. Intermediae Fomin, 1934, Fl. URSS 1: 21, p. p. - Subsect. Acrolysis Nakai, 1925, Bot. Mag. Tokyo, 39: 176.

Lectotype: $W$. polystichoides D. C. Eaton

Stipe apically obliquely articulated. Indusium membranaceous, reduced to a rim, hairy at margin.

About 5 species distributed in East Asia (W. macrochlaena Mett. ex Kuhn, W. oblonga Ching et $\mathrm{S} . \mathrm{H}$. Wu, W. polystichoides D. C. Eaton, W. pilosa Ching, W. subintermedia Tzvel.).

Sect. Subcordatae (Shmakov) Shmakov, comb. et stat. nov. - Ser. Subcordatae Shmakov, 1995, Surv. Fam. Woods. Eur.: 41.

Type: W. subcordata Turcz.

Stipe obliquely articulated above the middle. Indusium dissected onto filiform segmrnts enveloping sorus.

Highly polymorphic section; ca. 5 species distributed in East Asia (W. kitadakensis Ohwi, W. longifolia Tagawa, W. pseudoilvensis Tagawa, W. sinica Ching, $W$. subcordata Turcz.).

Subgen. Woodsia. - Sect. Woodsia sensu Ma, 1985, Fern Gaz. 13, 1: 23, p. min. p. - Sect. Euwoodsia Hook., 1844, Sp. Fil. 1: 63.

Type: $W$. ilvensis (L.) R. Br. 
Stipe with a transverse articulation below or above the middle. Lobes pubescent with hairs and scales.

Sect. Woodsia. - Sect. Euwoodsia Hook., 1844, Sp. Fil. 1: 63, pro subgen.

Type: W. ilvensis (L.) R. Br.

About 7 species distributed in the Northern Hemisphere (W. acuminata (Fomin) Sipl., $W$. asiatica Shmakov et Kiselev, W. calcarea (Fomin) Shmakov, $W$. gorovoii Krestsch. et Shmakov, $W$. ilvensis (L.) R. Br., W. pseudopolystichoides (Fomin) Kiselev et Shmakov, Woodsia taigischensis (Stepanov) Kuznetsov).

Sect. Alpinae (Shmakov) Shmakov comb. et. stat. nov. - Series Alpinae Shmakov, 1995, Surv. Fam. Woods. Eur.: 28.

Type: W. alpina (Bolt.) S. F. Gray

Four species, distributed in North America and mountains of Eurasia (W. alpina (Bolton) S. F. Gray, $W$. himalaica Ching et $\mathrm{S}$. K. Wu, $W$. intermedia Rupr. (W. gracilis (Lawson) Butters), W. pilosella Rupr.).

Subgen. Glabellae (Shmakov) Shmakov, comb. et stat. nov. - Woodsia sect. Woodsia subsect. Glabellae Shmakov, 1995, Surv. Fam. Woods. Eur.: 46.

Type: $W$. glabella R. Br.

Stipe with a transverse articulation below or above the middle. Fronds glabrous or pubescent with short glandules or with soft hairs at the lower side.

Sect. Glabellae (Shmakov) Tzvel., 2005, Novit. Syst. P1. Vasc., 37: 26. - Woodsia sect. Woodsia subsect. Glabellae Shmakov, 1995, Surv. Fam. Woods. Eur.: 46. - Subsect. Ilvensis Ching, 1932, Sinensia, 3, 5: 134, pro parte.

Type: $W$. glabella $\mathrm{R}$. Br.

About 7 species distributed in the Northern Hemisphere.

Series Glabellae Shmakov, 2003, Pteridol. New Millennium: 54. - Fronds glabrous.

Typus: $W$. glabella $\mathrm{R}$. Br.

Fronds glabrous.

About 5 species, distributed in the Northern Hemisphere (W. asplenioides Rupr., W. glabella R. Br., W. hancockii Bak., W. heterophylla (Turcz. ex Fomin) Shmakov, W. pinnatifida (Fomin) Shmakov).

Series Pulchellae Shmakov, ser. nov. - Fronds covered with short glands or at the lower side with soft hairs) - Plate operuit, cum brevibus glandulosis, sive molle fundo.
Type: $W$. pulchella Bertol.

Two species distributed in Europe and China (W. pulchella Bertol., W. shensiensis Ching).

Eriosoriopsis (Kitag.) Ching \& S. H. Wu, 1991, Fern Fam. \& Gen. China: 402.

Lectotype: E. rosthorniana Ching et $\mathrm{S}$. $\mathrm{H}$. Wu $(=$ Woodsia rosthorniana Deils $(=W$. jeholensis $\mathrm{Na}-$ kai et Kitag.))

Eight species distributed in the mountains of China, Himalayas and Taiwan.

Subgen. Eriosoriopsis - Woodsia sect. Eriosoriopsis Kitag., 1935, Rep. First. Sci. Expecl. Manchoukuo 4 (2): 48.

Lectotype: E. rosthorniana Ching et $\mathrm{S}$. $\mathrm{H}$. Wu (=Woodsia rosthorniana Deils $(=W$. jeholensis Nakai et Kitag.).

Sect. Eriosoriopsis - Woodsia sect. Eriosoriopsis Kitag., 1935, Rep. First. Sci. Expecl. Manchoukuo, 4 (2): 48. - Woodsia sect. Rostornia Tzvel. 2005, Novit. Syst. Pl. Vasc., 37: 38. - Woodsia subsect. Rosthornianae Shmakov et Kiselev, 1995, Surv. Fam. Woods. Eur.: 61.

Lectotype: E. rosthorniana Ching et S. H. Wu $(=$ Woodsia rosthorniana Deils $\quad(=W$. jeholensis Nakai et Kitag.)).

E. rosthorniana (Deils) Ching et S.H. Wu, 1991, Fern Fam. \& Gen. of China 402. - Woodsia rosthorniana Deils, 1900, in Engl. Bot. Jahrb. Syst. 29 (2): 187. - Type: "Plantae chinensis in prov. Setchuen ab insolis collectae, C. Bock \& A.V. Rosthorn (No. 34) 1764" (B).

E. guizhouensis (P. S. Wang, Q. Luo \& Li Bing Zhang) Shmakov, comb. nov. - Woodsia guizhouensis P. S. Wang, Q. Luo \& Li Bing Zhang, 2012, Novon, 22 (2): 191, fig. 1, 2. - Type: "China. Guizhou: Bijie City, Yangjiawang, Gonglongping Forestry Center, among crevices on wet limes tone cliff, $27^{\circ} 12.92^{\prime} \mathrm{N}, 104^{\circ} 59.52^{\prime} \mathrm{E}, 1670 \mathrm{~m}, 21 \mathrm{XI}$ 2008, Q. Luo 08301" (holotype - CDBI; isotypes $\mathrm{BJ}, \mathrm{MO})$.

Subgen. Eriosorus (Ching) Shmakov, comb. nov. - Woodsia subgen. Eriosorus (Ching) Shmakov, 2003, Pteridol. New Millennium: 59. - Woodsia sect. Eriosorus Ching, 1932, Sinensia 3 (5): 134.

Lectotype: E. lanosa (Hook.) Shmakov (Woodsia lanosa Hook.)

Sect. Eriosorus (Ching) Shmakov, comb. nov. - Woodsia sect. Eriosorus Ching, 1932, 
Sinensia 3 (5): 134. - Subsect. Eriosorus Shmakov et Kiselev, 1995, Surv. Fam. Woods. Eur.: 61.

Lectotype: E. lanosa (Hook.) Shmakov (Woodsia lanosa Hook.)

E. andersoni (Bedd.) Shmakov, comb. nov. Woodsia andersoni (Bedd.) Christ, 1905, Bull. Soc. Bot. France: Mem. 1: 45. - Gymnogramma andersoni Bedd., 1866, Ferns Brit. Ind. 2: 190, t. 190. Type: "Kumaon. T. Anderson" (K).

E. cinnamomea (Christ) Shmakov, comb. nov. Woodsia cinnamomea Christ, 1906, Bull. Acad. Int. Geogr. Bot. 16: 122. - Type: "Western China. E. H. Wilson, 5369, July 1903" (P; isotype - K).

E. cycloloba (Hand.-Mazz.) Shmakov, comb. nov. - Woodsia cycloloba Hand.-Mazz., 1929, Symb. Sinic. 6: 19, t. 1, f. 5. - Type: "NW - Yunnan: on granite and slate cliffs in Hg. St. of the Burmese Mountains under the Doker-la in the MekongSalwin chain, 28"15'. 4225 m., 17 Sept. 1915 H. Handel-Mazzetti, 8083" (W).

E. lanosa (Hook.) Shmakov, comb. nov. Woodsia lanosa Hook., 1868, Syn. Fil. 47. W. pellaeopsis Hand.-Mazz., 1929, Symb. Sinic. 6: 18, t. I, f. 6. - Type: "Pindari. Kumaon. Elevation above the sea 12000 feet, R. Strachey and J. E. Winterbottom, N 2" (K).

E. macrospora (C. Chr. et Maxon) Shmakov, comb. nov. - Woodsia macrospora C. Chr. et Maxon, 1927, J. Wash. Acad. Sci. 17(19): 499. Type: "Kansu: Taochow. Garganar Gorge, 36004200 m. alt. R. C. Ching 906. August. 29. 1923" (PE; isotype? - US).

E. okamotoi (Tagawa) Shmakov, comb. nov. Woodsia okamotoi Tagawa, 1938, Acta Phylotax. Geobot. (Kyoto) 7: 185. - Type: "Formosa. Prov. Takao: Mt. Kwanzan ca 3700 m. 7 Oct. 1937, S. Okamoto" (KYO).

\section{Key to the genera}

1. Stipes articulate

Woodsia

+ Stipes continuous

2

2. Scales unicolored

+ Scales bicolored with a darker middle part ....

\section{4}

3. Indusium spherical, more or less deeply, but not to the base, divided onto the blades

Physematium

+ Indusium almost to the base dissected onto the blades or narrow, usually filiform segments

Woodsiopsis

4. Indusia spherical, tearing when mature ...... 5

+ Indusium plate-like, dissected onto filiform lobes Eriosoriopsis

5. Lamina glabrous or with few glandular trichomes Protowoodsia

+ Lamina pubescent 6

6. Pinnae of first range attenuate and acute; sori not covered with recurved lobe dens

Hymenocystis

+ Pinnae of first range obtuse; sori slightly covered with recurved lobe dens .... Cheilanthopsis

Ключ для определения родов

1. Черешки с сочленением Woodsia

+ Черешки без сочленения ............................. 2

2. Чешуи одноцветные .................................... 3

+ Чешуи двуцветные, с более темной серединой

3. Индузии сферические, более или менее глубоко разорванные на лопасти, но не до основания Physematium

+ Индузии почти до основания рассечены на лопасти или на узкие, как правило, нитевидные сегменты

Woodsiopsis

4. Индузии сферические, разрывающиеся по созревании

+ Индузии пластинчатые, рассеченные на волосовидные доли Eriosoriopsis

5. Пластинки вай голые или с немногими железистыми волосками Protowoodsia

+ Пластинки вай опушенные 6

6. Доли первого порядка сужающиеся и заостренные; сорусы не прикрыты завернутым краем листа Hymenocystis

+ Доли первого порядка тупые; сорусы прикрыты завернутым краем листа

Cheilanthopsis

\section{LITERATURE}

Brown D.F.M. A monographic study of the fern genus Woodsia // Beihefte zur Nova Hedwigia, 1964. - Hf. 16. $154 \mathrm{~S}$.

Ching R.C. Study of Chinese Ferns VIII // Sinensia. - Nanking: China, 1932. - Vol. 3, No. 5. - P. 131-156.

Christenhusz M.J.M., Zhang X.-C., Schneider H. A linear sequence of extant families and genera of lycophytes and ferns // Phytotaxa, 2011. - Vol. 19. - P. 7-54.

Larsson A. Systematics of Woodsia. Ferns, bioinformatics and more. - Uppsala: Acta Universitatis Upsaliensis, 2014. - 36 p. (ISBN 978-91-554-9040-9). 
Ma Y.-L. Cytology and Taxonomy in Woodsiaceae. // Fern Gazette, 1985. - Vol. 13. - P. 17-24.

Nakai T. Notes on Japanese Ferns II // Bot. Magazine (Tokyo), 1925. - Vol. 39, No. 463. - P. 176-203.

Rothfels C.J., Sundue M.A., Kuо L.-Y., Larsson A., Kato M., Schuettpelz E., Pryer K.M. A revised family-level classification for eupolypod II ferns (Polypodiidae: Polypodiales) // Taxon, 2012. - Vol. 61, No. 3. - P. $515-533$.

Rothfels C.J., Larsson A., Kuo L.-Y., Korall P., Chiou W.-L., Pryer K.M. Overcoming deep roots, fast rates, and short internodes to resolve the ancient rapid radiation of eupolypod II ferns // Systematic Biology, 2012. - Vol. 61, No. 3. - P. 490-509.

Shmakov A.I. Key for the ferns of Russia. - Barnaul: Altai University Press, 1999. - 107 p.

Shmakov A.I. Synopsis of the ferns of Russia // Turczaninowia, 2001. - Vol. 4, No. 1-2. - P. 36-72.

Shmakov A.I. Review of the family Woodsiaceae (Diels) Herter of Eurasia // Pteridology in the New Millennium. Dordrecht - Boston - London: Kluwer Academic Publishers, 2003. - P. 9-64.

Shmakov A.I. Key for the ferns of Russia. - Barnaul: ARTIKA, 2009a. - 126 p.

Shmakov A.I. Synopsis of the ferns of North Asia // Turczaninowia, 2009b. - Vol. 12, No. 3-4. - P. 88-148.

Shmakov A.I. Ferns of North Asia. - Barnaul: ARTIKA, 2011. - 209 p.

Schmakov A.I., Kiselev A.Ya. A review of the family Woodsiaceae of Eurasia. - Barnaul: Altai State University Press, 1995. -89 p.

Tzvelev N.N. Polypodiophyta // Vascular plants of the Soviet Far East. - St. Petersburg, 1991. - Vol. 5. - P. 9-94.

Tzvelev N.N. De genera Woodsia R. Br. (Woodsiaceae, Polypodiophyta) // Novitates Systematicae Plantarum Vascularium. - St. Petersburg, 2005. - C. 33-46.

Zhang X.C., Kato M., Shmakov A. Woodsiaceae // Flora of China / Ed. by Z. Y. Wu, P. H. Raven \& D. Y. Hong. Vol. 2-3 (Pteridophytes). - Beijing: Science Press; St. Louis: Missouri Botanical Garden Press, 2013. - P. $397-404$. 